\title{
Description of a human Bocavirus recombinant strain in the Americas
}

\author{
Matías Salvo', Daiana Mir², Luis Fernando López Tort' \\ Andrés Lizasoain', Rodney Colina', Matías Victoria'1/+ \\ ${ }^{1}$ Universidad de la República, Centro Universitario Regional Litoral Norte, Laboratory of Molecular Virology, Salto, Uruguay \\ ${ }^{2}$ Universidad de la República, Centro Universitario Regional Litoral Norte, Genomics and Bioinformatics Unit, Salto, Uruguay
}

Human bocaviruses $(\mathrm{HBoV})$ are mainly associated with respiratory and gastroenteric infections. These viruses belong to the family Parvoviridae, genus Bocaparvovirus and are classified in four subtypes (HBoV1-4). Recombination and point mutation have been described as basis of parvovirus evolution. In this study three viral sequences were obtained from positives $\mathrm{HBoV}$ sewage samples collected in two Uruguayan cities and were characterised by different methods as recombinant strains. This recombination event was localised in the 5' end of VP1 gene and the parental strains belonged to subtypes 3 and 4 . These three Uruguayan strains are identical at the nucleotide sequences in the analysed genome region of the virus. As far as we known, this study represents the first detection of $\mathrm{HBoV}$ recombinants strains in the Americas.

Key words: human Bocavirus - recombinant - sewage - VP1 - Uruguay

Human Bocavirus (HBoV) belongs to the family Parvoviridae, genus Bocaparvovirus. Two species of primate Bocavirus compose the genus Bocaparvovirus: Primate Bocaparvovirus 1 and Primate Bocaparvovirus 2. ${ }^{(1)}$ In Primate Bocaparvovirus 1, two subtypes of the virus have been described: HBoV1 and HBoV3. HBoV1 was characterised in 2005 in pools of nasopharyngeal aspirates and was associated with respiratory tract infections, while $\mathrm{HBoV} 3$ was reported four years later in stool samples from children with acute gastroenteritis. ${ }^{(2,3)}$ In turn, subtypes $\mathrm{HBoV} 2$ and $\mathrm{HBoV} 4$ have been classified within Bocaparvovirus 2 being both associated with gastrointestinal infections. ${ }^{(3,4)}$

The HBoV genome consists of a single-stranded DNA which encodes three open reading frames (ORFs); ORFs 1 and 2 code the nonstructural proteins NS1 and NP1; and ORF3 codifies the capsid proteins VP1 and VP2. ${ }^{(2)}$ It is precisely in the 5' end of VP1 gene where the presence of a Bocavirus recombination hotspot has been evidenced. ${ }^{(4,5,6,7)}$ Although there is a solid evidence indicating intra-subtype recombination among $\mathrm{HBoV} 2$ variants at this recombination hotspot, there is still conflicting data regarding the suggested genesis of HBoV2 and $\mathrm{HBoV} 3$ via inter-subtype recombination occurring at this recombination point. ${ }^{(4,5,8,9,10)}$ Notably, it has been reported a trend without subtype distinction, of repeated VP1 gene replacement via recombination at this specific hotspot with $\mathrm{HBoV} 4$. This VP1 replacement would respond to the

doi: 10.1590/0074-02760190219

Financial support: Program Polo de Desarrollo Universitario, Universidad de la República, Uruguay; and project CSIC I+D 2010, UdelaR.

MS is an MSc student at Programa de Desarrollo de las Ciencias Básicas,

and has a scholarship from Agencia Nacional de Investigación e Innovación

- ANII, Uruguay.

+ Corresponding author: matvicmon@yahoo.com

(D) https://orcid.org/0000-0001-6101-6936

Received 27 June 2019

Accepted 08 October 2019 implicit advantage of borrowing the gene encoding the structural protein from the less prevalent $\mathrm{HBoV} 4$ subtype in order to overcome the host immunity.

A previous study performed by our group demonstrated a high frequency of $\mathrm{HBoV}$ in raw sewage from Uruguay; however, the presence of $\mathrm{HBoV}$ recombinant strains has not been reported so far in this country. In order to evaluate the presence of recombinant strains, three strains that could not be molecular characterised in that previous report were amplified in a larger genome fragment and sequenced. ${ }^{(11)}$

Samples were collected in the cities of Melo (CL12_12) and Treinta y Tres (TyT12_12, TyT2_13) between December 2012 and February 2013. Viruses were concentrated and the nucleic acid was extracted previously as described in Victoria et al. ${ }^{(12)}$ and Salvo et al. ${ }^{(11)}$ A nested polymerase chain reaction (nPCR) designed with forward primers described by La Rosa et al., ${ }^{(13)}$ and reverse primers described by Kapoor et al. ${ }^{(4)}$ was performed [PCR conditions and primers sequences are shown in Supplementary data (Tables I-II)]. First and second round PCR amplified a fragment of $911 \mathrm{bp}$ and $822 \mathrm{bp}$, respectively, corresponding to the 5' end of VP1 region of the HBoV genome (positions 2781 to 3692 and 2843 to 3665 of sequence FJ973562 for first and second round, respectively).

Amplicons were purified using PureLink ${ }^{\mathrm{TM}}$ Quick Gel Extraction kit and PCR Purification Combo kit (Invitrogen, Carlsbad, California, United States) and sequenced by Macrogen Platform (Seoul, South Korea) in an ABI3730XL Genetic Analyzer (Applied Biosystems, CA, USA) with forward and reverse primers of the second round PCR $(822 \mathrm{pb})$. Sequences were edited with SeqMan Software (DNAstar Lasergene) and aligned by using MUSCLE program along with $\mathrm{HBoV}$ reference sequences retrieved from the NCBI Database. ${ }^{(14)}$

Sequences obtained were submitted to a nucleotide based Basic Local Alignment Search Tool (BLASTn) analysis in order to recover $\mathrm{HBoV}$ sequences presenting high nucleotide similarity and probably a similar recombination profile. ${ }^{(15)}$ To further determine the potential recombination events, sequences were fragmented and 
the regions that stretch upstream and downstream the recombination breakpoint described in a Russian recombinant sequence (the most similar sequence), were submitted to a Maximum Likelihood (ML) phylogenetic analysis implemented in the IQ-TREE software along with reference $\mathrm{HBoV}$ sequences. ${ }^{(16)}$ The most appropriate evolutionary model for each partial genome alignment was determined by ModelGenerator v.0.851. ${ }^{(17)}$ The phylogenetic trees were visualised with FigTree v1.4.3 (http://tree.bio.ed.ac.uk/software/figtree/) and the robustness of each node was assessed by an ultrafast bootstrap approximation (1000 replicates). ${ }^{(18)}$

To confirm the results, Similarity and Bootscan analyses as implemented in Simplot v3.5.1 and RDP4 programs, respectively, were carried out. ${ }^{(19,20,21)}$ Similarity plot analyses were conducted using a $200 \mathrm{bp}$ sliding window and $20 \mathrm{bp}$ step size. Bootscan analyses were implemented using a window size of $150 \mathrm{bp}$; step of 20 bp and pairwise distance with 100 bootstrap replicates.

The $\mathrm{HBoV}$ sequences generated in this study were deposited in the GenBank database under the following accession numbers: MK442008 to MK442010.

The three evaluated Uruguayan sequences showed $100 \%$ nucleotide identity in the evaluated region, and retrieved the Russian recombinant strain RUS_NSC_11-
N2512 (GenBank accession number: KJ710645) isolated in 2011 as its top Blast hit (e-value: 0.0; Identity: 99.0). This Russian sequence has already been described as a recombinant strain between $\mathrm{HBoV} 3$ and $\mathrm{HBoV} 4$ subtypes. ${ }^{(7)}$

As it can be seen in the phylogenetic reconstruction based on the genomic region upstream to the recombination point (Fig. 1), the Uruguayan sequences comprise a cluster with sequences belonging to the $\mathrm{HBoV} 3$ subtype (Fig. 1A). Alternatively, in the inferred phylogenetic tree based on the sequence region downstream to the recombination point, the evaluated Uruguayan sequences group within the HBoV4 monophyletic clade (Fig. 1B).

To confirm the results obtained by the phylogenetic analyses, similarity (Fig. 2A) and bootscan analyses (Fig. 2B) were performed. Plots predicted a recombination site located nearby the nucleotide 200 (cutoff, $70 \%$ ) of the Uruguayan HBoV strains. The predicted site for the recombination break-point roughly corresponds to the nucleotide position 3,250 of the $\mathrm{HBoV}$ complete genome of the recombinant strain RUS_NSC_11-N2512; thus, it was located at the 5' end of the VP1 gene. The recombination profile suggests that the 5' end of the evaluated VP1 region of the Uruguayan sequences is a $\mathrm{HBoV} 3$ subtype, while the 3' end downstream the recombination point belongs to the $\mathrm{HBoV} 4$ subtype.
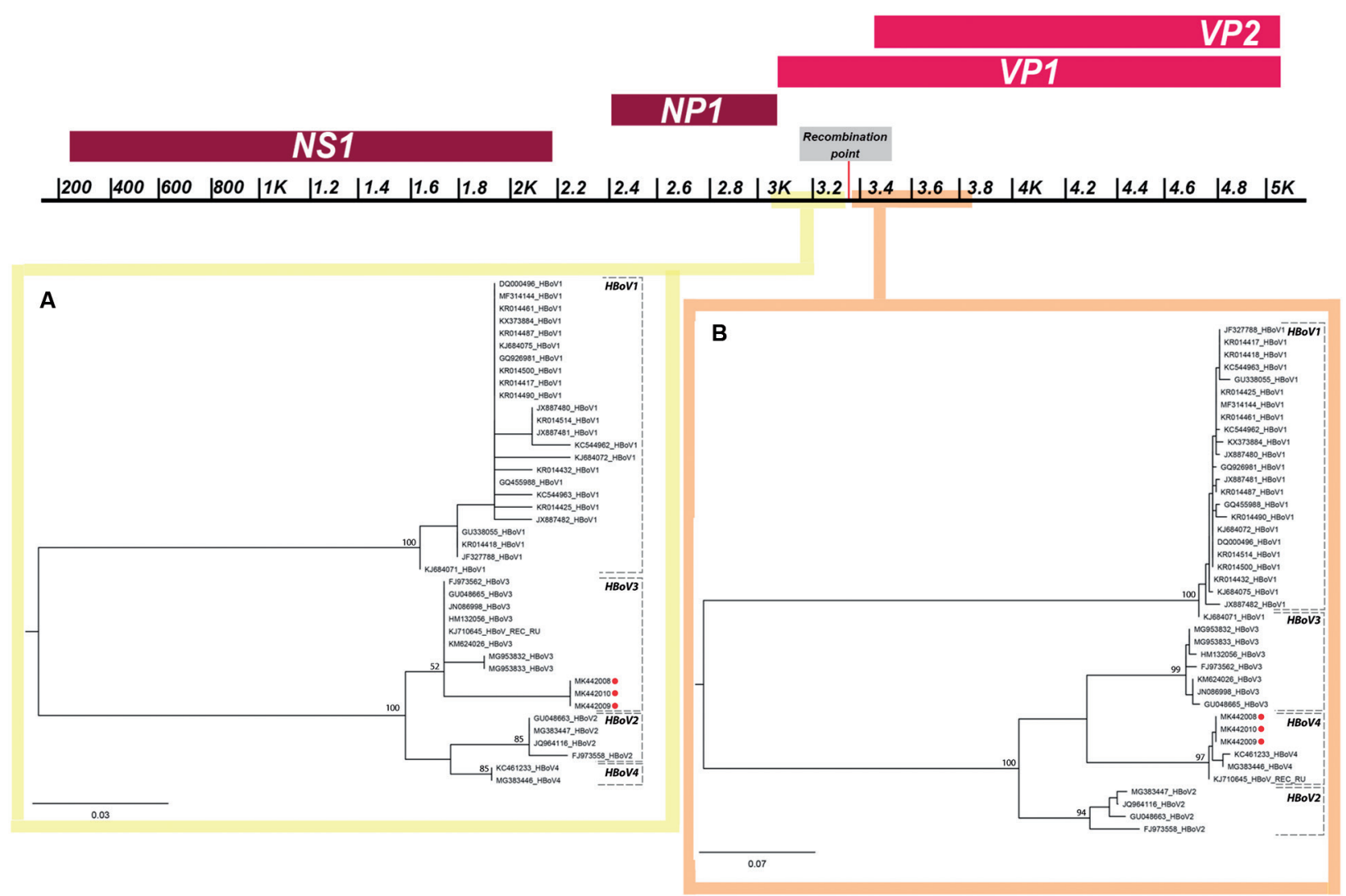

Fig. 1: maximum likelihood phylogenetic analysis of human Bocavirus (HBoV) strains under the HKY nucleotide substitution model. The results obtained using the VP1 region upstream (120 bp) and downstream (490 bp) to the recombination point are shown in A and B, respectively. The three Uruguayan strains are indicated by red squares. Bootstrap support values are indicated at key nodes. The branch lengths are drawn to scale with the bar at the bottom indicating nucleotide substitutions per site. A map of the HBoV genome highlighting the analysed regions and the inferred position of the recombination point is shown at the top. 
A

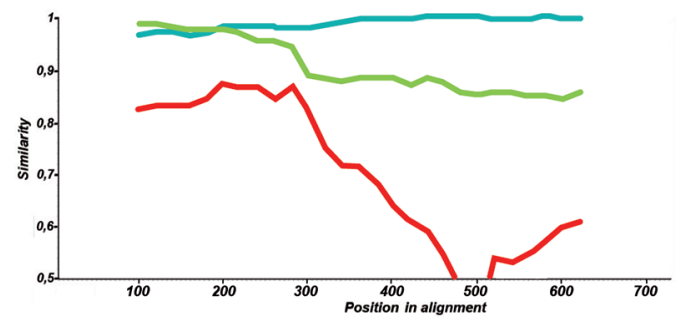

B

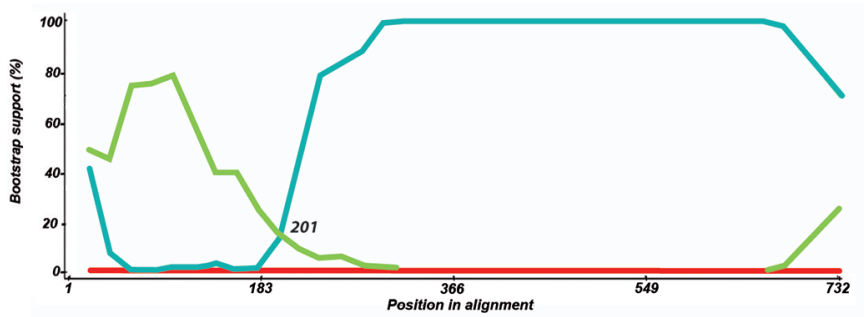

References: —KC461233_HBoV4 FJ973562_HBoV3 —GU338055_HBoV1

Fig. 2: similarity (A) and bootscan (B) analyses of query Uruguayan sequence MK442008. In the similarity plot analyses, the y-axis represents the percentage of genetic identity, per analysis window, for the query strain with human Bocavirus (HBoV) reference strains. The bootscan plots depict the percentage (bootstrap values) at which each reference strain co-segregates phylogenetically, in the analysis window, with the query strain. Reference HBoV strains are represented by a colour code as indicated at the bottom of the figure.

As far as we known, this is the first description of recombinant strains of $\mathrm{HBoV}$ detected in the Americas. These strains, which were analysed in a partial region of the VP1 variable gene, showed a high nucleotide similarity $(99 \%)$ with the recombinant sequence described in Russia. ${ }^{(7)}$ The Uruguayan sequences corresponded to recombinant strains between the parental subtypes 3 and 4 of $\mathrm{HBoV}$ and the break-point is in a region already described as a hotspot recombination in Parvovirus. ${ }^{(22)}$ Following this strategy the virus could generate genetic variation and adaptive advantages, especially in overcoming host immunity, as it was already discussed by Tyumentsev et al. ${ }^{(7)}$

In our previous study, $\mathrm{HBoV}$ sequences were obtained in $70 \%$ of the positive sewage samples and three of them (9\%) could not be classified in any of the HBoV subtypes. ${ }^{(1)}$ In the present study, these strains were classified as recombinant strain using a longer genome fragment including the recombination point. The fact that three samples with the same recombinant sequence were detected in sewage samples of different cities and in different periods of the year, suggests that the recombinant strain has dispersed and successfully replicated in their host acquiring possible immunological advantages over the parental strains.

It is important to remark the suitability of using the primers described by La Rosa et al.. ${ }^{(13)}$ for the phylogenetic characterisation. Although recombinant sequences cannot be associated to a particular clade of the subtypes already described since the hotspot recombination is located approximately in the middle of the amplified sequence, this protocol, can be used as a first approach in order to identify recombinant strains. Moreover, the combination of primers described by La Rosa et al. ${ }^{(13)}$ and Kapoor et al. ${ }^{(4)}$ is an adequate approach in order to confirm $\mathrm{HBoV}$ recombinant strains when the recombination point is located at the 5' end of the VP1 gene.

More studies are needed in order to identify this recombinant strains in human clinical samples and evaluate if they are more diverse with respect to others subtypes and also, if these recombinant strains generate a more virulent clinical outcome in human hosts. Unfor- tunately, in our study we did not have access to stools from children presenting acute gastroenteritis in the same cities where the recombinant strains were found in order to confirm the presence of this recombinant strain in symptomatic patients.

Recombination events seem to be an important evolutionary force that shapes the $\mathrm{HBoV}$ genome. In the near feature, with more results of evolutionary studies on $\mathrm{HBoV}$, the origin of each $\mathrm{HBoV}$ subtypes as well as the different circulating recombinant strains, their classification and clinical outcome will be elucidated.

\section{ACKNOWLEDGEMENTS}

To the OSE, Uruguay, for their technical assistance.

\section{AUTHORS' CONTRIBUTION}

MS, DM, LFLT and AL performed the experiments and the bioinformatics analysis; MS and DM wrote the manuscript; RC and MV designed the study and critically analysed the results and the manuscript.

\section{REFERENCES}

1. ICTV, Virus Taxonomy. 2016 Release. Available from: https://talk. ictvonline.org/taxonomy/.

2. Allander T, Tammi MT, Eriksson M, Bjerkner A, Lindell AT, Andersson B. Cloning of a human parvovirus by molecular screening of respiratory tract samples. Proc Natl Acad Sci USA. 2005; 102(36): 12891-6.

3. Arthur JL, Higgins GD, Davidson GP, Givney RC, Ratcliff RM. A novel bocavirus associated with acute gastroenteritis in Australian children. PLoS Pathog. 2009; 5(4): e1000391.

4. Kapoor A, Simmonds P, Slikas E, Li L, Bodhidatta L, Sethabutr $\mathrm{O}$, et al. Human bocaviruses are highly diverse, dispersed, recombination prone, and prevalent in enteric infections. J Infect Dis. 2010; 201(11): 1633-43.

5. Fu X, Wang X, Ni B, Shen H, Wang H, Zhang X, et al. Recombination analysis based on the complete genome of bocavirus. Virol J. 2011; 8(12): 182.

6. Khamrin P, Okitsu S, Ushijima H, Maneekarn N. Complete genome sequence analysis of novel human bocavirus reveals genetic recombination between human bocavirus 2 and human bocavirus 4. Infect Genet Evol. 2013; 17(7): 132-6. 
7. Tyumentsev AI, Tikunova NV, Tikunov AY, Babkin IV. Recombination in the evolution of human bocavirus. Infect Genet Evol. 2014; 28(12): 11-14.

8. Kapoor A, Slikas E, Simmonds P, Chieochansin T, Naeem A, Shaukat $\mathrm{S}$, et al. A newly identified bocavirus species in human stool. J Infect Dis. 2009; 199(2): 196-200.

9. Cheng W, Chen J, Xu Z, Yu J, Huang C, Jin M, et al. Phylogenetic and recombination analysis of human bocavirus 2. BMC Infect Dis. 2011; 11: 50.

10. Chong YL, Ng KH. Genomic recombination in primate bocavirus: inconsistency and alternative interpretations. Virus Genes. 2017; 53(6): 774-7.

11. Salvo M, Lizasoain A, Castells M, Bortagaray V, Castro S, Colina $\mathrm{R}$, et al. Human bocavirus: detection, quantification and molecular characterization in sewage and surface waters in Uruguay. Food Environ Virol. 2018; 10(2): 193-200.

12. Victoria M, Tort LF, García M, Lizasoain A, Maya L, Leite JP, et al. Assessment of gastroenteric viruses from wastewater directly discharged into Uruguay River, Uruguay. Food Environ Virol. 2014; 6(2): 116-24.

13. La Rosa G, Della Libera S, Iaconelli M, Donia D, Cenko F, Xhelilaj G, et al. Human bocavirus in children with acute gastroenteritis in Albania. J Med Virol. 2015; 88(5): 906-10.

14. Edgard RC. MUSCLE: multiple sequence alignment with high accuracy and high throughput. Nucleic Acids Res. 2004; 32(5): 1792-7.
15. Altschul SF, Gish W, Miller W, Myers EW, Lipman DJ. Basic local alignment search tool. J Mol Biol. 1990; 215(3): 403-10.

16. Nguyen LT, Schmidt HA, Von Haeseler A, Minh BQ. IQ-TREE: a fast and effective stochastic algorithm for estimating maximum likelihood phylogenies. Mol Biol Evol. 2015; 32(1): 268-74.

17. Keane TM, Creevey CJ, Pentony MM, Naughton TJ, Mclnerney JO. Assessment of methods for amino acid matrix selection and their use on empirical data shows that ad hoc assumptions for choice of matrix are not justified. BMC Evol Biol. 2006; 6(3): 29.

18. Minh BQ, Nguyen MAT, Von Haeseler A. Ultrafast approximation for phylogenetic bootstrap. Mol Biol Evol. 2013; 30(5): 1188-95.

19. Salminen M, Carr JK, Burke DS, Mc Cutchan FE. Identification of breakpoints in intergenotypic recombinants of HIV type 1 by bootscaning. AIDS Res Hum Retrovirus. 1995; 11(11): 1423-5.

20. Lole KS, Bollinger RC, Paranjape RS, Gadkari D, Kulkarni SS, Novak NG, et al. Full-length human immunodeficiency virus type 1 genomes from subtype C-infected seroconverters in India, with evidence of intersubtype recombination. J Virol. 1999; 73(1): 152-60.

21. Martin DP, Murrell B, Golden M, Khoosal A, Muhire B. RDP4: detection and analysis of recombination patterns in virus genomes. Virus Evol. 2015; 1(1): vev003.

22. Lefeuvre P, Lett JM, Varsani A, Martin DP. Widely conserved recombination patterns among single-stranded DNA viruses. J Virol. 2009; 83(6): 2697-707. 